\begin{tabular}{c} 
journal homepage: http://ijiemjournal.uns.ac.rs/ \\
International Journal of Industrial \\
Engineering and Management \\
Volume $11 /$ No $1 /$ March $2020 / 31-39$ \\
\hline
\end{tabular}

Original research article

\title{
Model of Human Error Probability based on dual-phase approach for learning process in cognitive-oriented tasks
}

\author{
F. Facchini *, S. Digiesi, G. Mummolo \\ Department of Mechanics, Mathematics and Management, \\ Polytechnic University of Bari, Bari, Italy
}

\section{A B STRACT}

Human Error Probability (HEP) is a key element in the chain of events that could lead up to system accidents and despite the efforts of recently studies to evaluate the human reliability, many of the limitations and problems have not yet been solved. The model proposed tries to overcome these limitations by means of a method that combines the advantages of a dual-phase learning approach with that of a multi-attribute utility analysis, in accordance with Dar-El's theory. Results of numerical simulations show the effectiveness of the model in quantifying, over time, the HEP and in evaluating the human task error proneness by varying the work breaks schedule.

\section{Introduction}

New accidents or incidents due to human error do not "just happen" and in most of the cases the cause relies not only on human incompetence or negligence [1]. In scientific literature the Human Error Probability (HEP) is defined, in case of discrete tasks, as the number of errors divided by the number of opportunities for making errors. The HEP is a key element in system accidents and disasters, especially in high-risk fields, such as industrial plants, nuclear plants, and aerospace. According to Health and Safety Executive agency (HSE), the errors can be classified in two categories. The first category includes the
ARTICLE INFO

Article history:

Received August 28, 2019

Revised January 21, 2020

Accepted January 27, 2020

Published online February 3, 2020

Keywords:

Human Error Probability;

Human Reliability Assessment;

Fatigue-Recovery;

Learning-Forgetting;

Work Breaks Schedules

*Corresponding author:

Francesco Facchini

francesco.facchini@poliba.it 
the worker does not know the correct way of carrying out a task either because it is a new task or because he is not properly trained, or both [2]. Recent studies showed that in industrial sectors more than $80 \%$ of defects due to assembly and visual inspection tasks depend on human behaviour [3]. The human errors can be the root cause of serious individual injuries and catastrophic accidents in industry as well as in urban areas [4]. According to Purpura (2013), the human error and unsafe behaviour accounts for $90 \%$ of all accidents in work environment [5]. Last report on aviation safety performance, published by the International Air Transport Association, shows that most of the airplane accidents (approximately the $80 \%$ ) are due to human errors (e.g. pilots, air traffic controllers, mechanics, etc.) and only the $20 \%$ percent are due to machine failures [6]. The IChemE

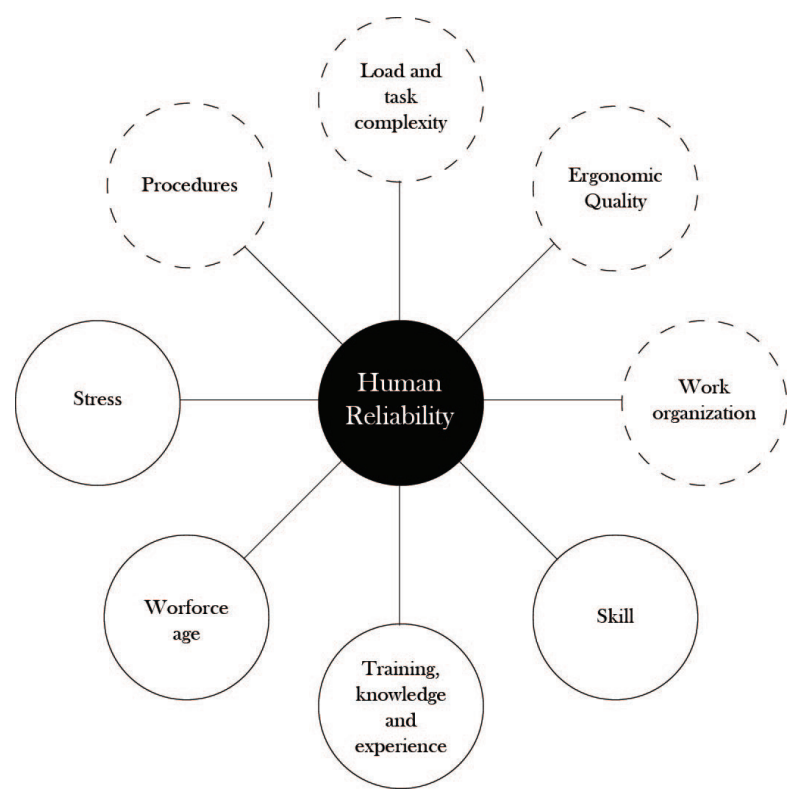

Figure 1. External (---) and Internal (- - ) factors that affect the human reliability
Loss Prevention Bulletin identified sixteen different causes (table 1), related to workers behaviour, that can originate an accident in workplaces. Consistently whit the classification provided, the report accounts almost $50 \%$ of the examined accidents originated by "poor concentration, inattention, fatigue" (see tab. 1) and approximately $15 \%$ by unsafe worker operation and psychological causes [7]. Therefore, high human reliability is considered essential for ensuring the safe and reliable operation of complex systems as well as to ensure proper organizational and management strategy [8]. The Human Reliability (HR) is defined as the probability that an individual, a team or a human organization accomplishes an activity under variable constraints, due to different circumstances, within a certain period of time [9].

There are many internal and external factors able to affect the human reliability (fig. 1): internal factors are related to characteristics of the individual worker such as training, knowledge, experience as well as the age of the worker [10]. External factors affecting the human reliability include all aspects related to the typology of the tasks carried out (e.g. procedures, load and task complexity, etc.) as well as the work environment characteristics (e.g. ergonomics, work organization, etc.) [11].

The Human Reliability Assessment (HRA) allows, on the basis of the workers behaviour and of the work environment, to evaluate the occurrence probability of human-related failure events in the designed scenarios. Traditional HRA methods are based on different approaches applicable in different industrial tasks, according to the typology of tasks to be performed (physical or cognitive oriented), level of tasks-complexity, operator capabilities as well as workplace conditions.

Table 1. Cause of accident in workplace

\begin{tabular}{|c|c|c|c|}
\hline ID & Cause & ID & Cause \\
\hline \#01 & Technical Deficiency & \#09 & Poor training/instruction \\
\hline \#02 & Design Error & $\# 10$ & Poor communication / co-ordination \\
\hline$\# 03$ & Change which was not thoroughly examined & \#11 & Decision of superiors concerning technical matters \\
\hline$\# 04$ & Unforeseen, unknown & $\# 12$ & Decision of superiors concerning organization \\
\hline \#05 & Worker/Operator works in an unsafe manner & \#13 & Physiological, psychological causes \\
\hline \#06 & Toleration by the supervisor & $\# 14$ & Poor concentration, inattention, fatigue \\
\hline$\# 07$ & Lack of supervision & \#15 & Remaining risk \\
\hline \#08 & No use or improper use of work permits & \#16 & Unknown or undetermined causes \\
\hline
\end{tabular}


The existing scientific literature offers a wide range of statistical models for analysing and predicting the human reliability. According to Calixto et al. (2013), the HRA methods can be classified in three main groups: the first (1970-1990) is known as the first human reliability methods generation, which focuses on human error probabilities and operational human errors, by considering the worker like a mechanical component [12]. In most of these methods, such as Technique for Human Error Rate Prediction (THERP), Human Error Assessment and Reduction Technique (HEART), and Human Cognition Reliability (HCR), the HEP is evaluated on the basis of the characteristics of the task assigned to the operator, and its value is obtained in accordance with the Performance Shaping Factors (PSF), quantifying the impact on the human performance of environmental factors [13]. The second human reliability methods generation (1990-2005) is focused on the human performance considering the cognitive processes based on factors like workload, stress, sociological issues, psychological issues, illness, etc. Under this perspective, the second generation methods, such as A Technique for Human Error Analysis (ATHEANA), the Cognitive Reliability and Error Analysis Method (CREAM), and the Méthode d' Evaluation de la Realisation des Missions Operateur pour la Surete (MERMOS) provide a qualitative evaluation of the operator's behaviour in an integrated system MenTechnology-Organisation [14]. Finally, the third human reliability methods generation (from 2005 until today) includes methods focused on the human performance in relations with an artificial work environment (e.g. Probabilistic Cognitive Simulator for HRA Studies (PROCOS), Connectionism Assessment of Human Reliability (CAHR), etc.). These methods provide a dynamic model that allows reproducing the human decisions and actions in work environment by means of simulation (virtual environment and virtual performers) and/or simulators of virtual environment with human performers [15].

In scientific literature several studies are available focusing on the application of the first, second, and third generation methods. Zhang, et al. (2019) developed a dynamic human reliability assessment approach, known as Predicted Mean Vote-Cognitive Reliability and Error Analysis Method (PMVCREAM), aiming at evaluating the cognitive actions of oceanauts during the procedures required by deep-sea missions. The results showed that the model allows to estimate the Cognitive Failure Probability (CFP) in order to avoid human error in deep-sea research, thereby preventing injury and loss of life during undersea job [16]. Shirali et al. (2019) adopted a CREAM Bayesian Network (BN) approach in a control room of a petrochemical plant in order to identify the HEP and to overcome the limitations due to variable relationship between operator and control mode [17]. An HRA model in rail transport field has been developed in order to identify the specific risk due to repetitive tasks taking into account the data uncertainty [18, 19]. Starting from this model, the authors developed a specific PSF able to support the HEP identification in railway operations [18]. Margiotta et al. (2016) consider the human reliability one of the most important elements for evaluating the human performance, and at this aim introduce innovative wearable wireless systems of control to be adopted [20]. A model based on Dynamic Bayesian approach has been developed by Abaei et al. (2019) to evaluate the HR in daily tasks in offshore operations, and results obtained showed that the HEP in marine operations increases towards the end of operational days, and the high variation in human reliability is mainly dependent on weather conditions [21]. A new methodology based on revised HEART has been developed by Islam et al. (2017) to assess and quantify the potential human errors in different marine environmental and operational conditions. The application of the developed methodology on the maintenance procedures of a marine engine exhaust turbocharger on an offshore oil and gas facilities confirms that extreme weather, extreme workplace temperature, high ship motion, high level of noise and vibration and work overload and stress increase the likelihood of human error as well as of potential accidents [22]. In 2017, Falcone et al. introduced a hybrid model that integrates the advantages of the methodologies HEART, Standardized Plant Analysis Risk-Human (SPAR-H) and Success Likelihood Index Method (SLIM) in order to evaluate the impact of all environmental and behavioural factors on the decisions and the actions of operators in case of accidents and disasters. Results obtained from the analysis of a real case study provide an empirical and a theoretical contribution referring to the framework used to detect human error, evaluating the workers' reliability under emergency conditions [23]. A model for assessing the probability of human errors in reconfigurable manufacturing systems based on tasks characteristics, work environment as well as workers capabilities has been developed using the multi-attribute utility analysis by Elmaraghy et al. (2008). The developed model has been validated in a real industrial case. The multi-attribute utility analysis, on the basis of the Elmaraghy's model, evaluates the HEP 
independently of time. The attributes considered allow identifying the HEP neglecting the effect due to 'time-dependent' phenomena like fatigue, forgetting, learning, etc [24]. Consistently with this lack, Givi et al. (2015) introduced a Learning-Forgetting-Fatigue-Recovery Model (LFFRM) where the HEP depends on two 'time-dependent' utility functions [25]:

(1) Normalised Fatigue Score (NFS), related to the accumulated fatigue over time (F) and considering the residual fatigue after a break (R);

(2) Normalised Learning Score (NLS), given by the rate between the time required to perform the n-th repetition of a given task (tx) and the time required to perform the first repetition of the same task $\left(\mathrm{T}_{1}\right)$.

In scientific literature, starting from the work of Wright (eq. 1) [26] many models have been defined in order to describe the dynamic variability of the task completion time due to the learning effects.

$$
\mathrm{t}_{\mathrm{X}}=\mathrm{T}_{1} \cdot \mathrm{x}^{\frac{\log (\mathrm{LR})}{\log (2)}}
$$

where $\mathrm{x}$ is the cumulative number of tasks completed and LR identifies the learning rate $(0<\mathrm{LR}<1)$. With the increase of the LR-value it is reduced the workers' learning ability.

According to Dar-El et al. (1995) [27] there is evidence that the model of Wright tends to underestimate the execution time of a manual repetitive task when early data are adopted, and when execution time data related to a relevant number of tasks completed are considered, the estimates obtained for the performance at the first repetition, $y(1)$, and for the LR-value tend to be underestimated. The Authors addressed this discrepancy to the dual nature of each task, jointly consisting of a cognitive and a motor part, both subjected to the learning phenomenon. Cognitive and motor learning affect workers behaviour in different time period during the work shift and with different magnitude, so as the resulting reduction of the task completion times cannot be described by means of a single and constant (over the work shift) LR. Therefore, a dual-phase learning model is introduced in order to consider both cognitive and motor aspect related to the task under investigation. The dual-phase model considers that in early stages of learning, the operator mainly uses cognitive skill to perform the task correctly, i.e. following instructions, remembering the sequence of operations, developing the correct method, etc. As experience is gained in executing the task, the time spent on cognitive activity is sharply reduced and the performance is dominated by motor learning. According to considerations made for dual-phase learning model, it is possible to claim that the observed curve is given by the sum of a cognitive and a motor learning curves.Consistently with this approach, $\mathrm{t}_{\mathrm{x}}$ depends on the times required for the first cycle under cognitive $\left(\mathrm{t}_{\mathrm{c}}{ }^{(1)}\right)$ and motor $\left(t_{m}{ }^{(1)}\right)$ conditions and by learning constants under cognitive $\left(b_{c}\right)$ and motor conditions $\left(b_{m}\right)$, as showed in eq. 2.

$$
\mathrm{t}_{\mathrm{x}}=\mathrm{t}_{\mathrm{c}}^{(1)} \cdot \mathrm{x}^{-\mathrm{b}_{\mathrm{c}}}+\mathrm{t}_{\mathrm{m}}^{(1)} \cdot \mathrm{x}^{-\mathrm{b}_{\mathrm{m}}}
$$

Cognitive learning proves to affect workers behaviour in the early cycles and with a higher magnitude of that of motor learning, which becomes predominant for large number of repetitions. As a consequence, in case of manual task (low cognitive content task- e.g. push a button, tighten and unscrew the same screws, plug-in the same connections, etc.) a low reduction of task completion time $\left(\mathrm{t}_{\mathrm{x}}\right)$ with the increase of the number of tasks completed is observed. The results of the study are quite intuitive: indeed, it is very hard to reduce over time a repetitive task characterized mainly from physical exertions. On the contrary, the completion time of a task with a high cognitive content is subjected to high reduction, since it does not depend on manual movements hard to be optimized beyond certain limits with learning and it is less subjected to muscle fatigue.

In absence of experimental data and considering a workload mainly characterized by cognitive-oriented repetitive tasks (e.g. visual inspection, signal recognition and interpretation, complex object manipulation, etc.) the dual-phase model provides a reliable estimation of $t_{x}$ if compared to other available models.

Although on the basis of the literature review conducted it is possible to claim that there are different approaches available allowing to provide a good guide on how to evaluate the human reliability, there are still aspects to be discussed in-depth. In particular three main aspects required further investigations:

(1) the existing approaches are not capable to predict when the error occurs, considering the impact due to possible changes over time (e.g. physical and mental fatigue, work break schedule, cognitive workloads, etc.);

(2) the existing models allows to evaluate the HEP according to tasks characteristics, work 
environment and workers capabilities, neglecting the effects due to 'time-dependent' aspects; in the Learning-ForgettingFatigue-Recovery these effects are considered, but independently from tasks characteristics, work environment, and workers capabilities;

(3) the available models are hard to be implemented in different contexts.

The model proposed in this paper tries to overcome these limitations in case of cognitive-oriented tasks by means of a method that combines the advantages of the dual-phase approach with the advantages of a multi-attribute utility analysis. A model in accordance with the dual-phase approach proposed by [26] allowing to estimate dynamic variability of the HEP over time has been developed. In the model, the effects due to fatigue and of recovery as well as of learning and forgetting are considered.

The mathematical model developed on the basis of multi-attribute analysis proposed by [24] allows to identify the HEP at the beginning of the work shift $(\mathrm{t}=0)$. Starting from HEP value at $\mathrm{t}=0$ and considering the work environment, the task complexity, and the worker skill level as well as the work break schedule, the model allows identifying the dynamic variability of the HEP over time.

The remainder of this paper is organized as follows: the model is introduced in section 2; discussion of results obtained by applying the model to three different work rest schedules are in section 3; conclusions of this work are in section 4 .

\section{Methodology and Model application}

Notation adopted in the model is listed below:

$\operatorname{HEP}(\mathrm{t}), \operatorname{HEP}(0)$

$\mathrm{U}(\mathrm{t}), \mathrm{U}(0)$

$\mathrm{u}_{\mathrm{x}}, \mathrm{u}_{\mathrm{y}}, \mathrm{u}_{\mathrm{z}}$

$\mathrm{w}_{\mathrm{x}}, \mathrm{w}_{\mathrm{y}}, \mathrm{w}_{\mathrm{z}}$

for time $t>0$ and $t=0$, respectively;

human error utility function for time $t>0$ and $\mathrm{t}=0$, respectively;

utility functions due to task error proneness (x), worker characteristics (y), and work environment (z);

\begin{tabular}{|c|c|}
\hline NLS, NFS & learning \\
\hline & $\begin{array}{l}\text { and fatigue score, } \\
\text { respectively; }\end{array}$ \\
\hline$w_{l}, w_{f}$ & $\begin{array}{l}\text { relative weights of NLS } \\
\text { and NFS }\left(w_{l^{+}} w_{f}=1\right) ;\end{array}$ \\
\hline$\alpha, \beta$ & $\begin{array}{l}\text { constants multiplier as } \\
\text { defined in (Givi et al., } \\
\text { 2015); }\end{array}$ \\
\hline $\mathrm{t}_{\mathrm{x}}, \mathrm{T}_{1}$ & $\begin{array}{l}\text { time required to perform } \\
\text { the n-th repetition of a } \\
\text { task and the first } \\
\text { repetition of the same } \\
\text { task, respectively; }\end{array}$ \\
\hline $\mathrm{F}(\mathrm{t}), \mathrm{F}_{\max }$ & $\begin{array}{l}\text { accumulated fatigue over } \\
\text { time and maximum } \\
\text { accumulated } \\
\text { respectively; }\end{array}$ \\
\hline $\mathrm{R}$ & $\begin{array}{l}\text { residual fatigue } \\
\text { break; }\end{array}$ \\
\hline$\lambda$ & $\begin{array}{l}\text { severity index of the } \\
\text { work performed }(0-1 \\
\text { ranging); }\end{array}$ \\
\hline$\mu$ & $\begin{array}{ll}\text { worker's recovery rate } \\
\text { index }(0-1 \text { ranging }) ;\end{array}$ \\
\hline$\tau$ & length of the break. \\
\hline
\end{tabular}

The model developed allows identifying the HEP over time $(\operatorname{HEP}(t))$ on the basis of the following approaches: the multi-attribute utility analysis, introduced by Elmaraghy et al. (2008), evaluates the $\mathrm{HEP}(0)$ considering the time-independent aspects. In this case, the $\operatorname{HEP}(0)$ depends on human error utility function (eq. 3) where $\mathrm{U}(0)$ is the weighted average of the overall utility functions $\mathrm{u}_{\mathrm{x}}, \mathrm{u}_{\mathrm{y}}, \mathrm{u}_{\mathrm{z}}$ (eq. 4). Each utility function is obtained through an in-depth assessment of the task error proneness (e.g. difficulty, diversity/coupling between task/s, etc.), worker(s) characteristics (i.e. professional and personal capabilities) as well as work environment (e.g. physical work environment, frequency of reconfiguration, etc.). More details on the evaluation procedure can be found in [24].

$$
\begin{aligned}
& \operatorname{HEP}(0)=10 \mathrm{E}(6 \log (\mathrm{U}(0))) \\
& \mathrm{U}(0)=\mathrm{w}_{\mathrm{x}} \cdot \mathrm{u}_{\mathrm{x}}+\mathrm{w}_{\mathrm{y}} \cdot \mathrm{u}_{\mathrm{x}}+\mathrm{w}_{\mathrm{y}} \cdot \mathrm{u}_{\mathrm{x}}
\end{aligned}
$$

Givi et al. (2015) evaluated the human error utility function over time by means of the Learning-Forgetting-Fatigue-Recovery Model (LFFRM) [25]; this approach is mainly focused on time-dependent aspects, consistent with the following equations: 


$$
\begin{aligned}
& \mathrm{U}(\mathrm{t})=\mathrm{w}_{\mathrm{l}} \cdot \mathrm{NLS} \cdot \alpha+\mathrm{w}_{\mathrm{f}} \cdot \mathrm{NFS} \beta \\
& \mathrm{NLS}=\frac{\mathrm{F}(\mathrm{t})}{\mathrm{F}_{\max }} \\
& \mathrm{NFS}=\frac{\mathrm{t}_{\mathrm{x}}}{\mathrm{T}_{1}}
\end{aligned}
$$

The dual-phase approach for learning process in cognitive-oriented tasks developed is based on the jointly adoption of the multi-attribute utility analysis and of the LFFRM model. The model assumes that for task characterized by a predominant cognitive activity with cycle time less than 5 minutes, the time required to perform the first repetition of the task can be considered $t_{x}=T_{1}$. Under these assumptions, the accumulated fatigue $(\mathrm{F}(\mathrm{t}))$ at the beginning of the work shift $(\mathrm{t}=0)$ can be considered null. Therefore, in accordance with equations 6 and 7 , the values of NLS and NFS will be 1 and 0, respectively. The $\mathrm{U}(0)$ evaluated under these conditions through the multi-attribute utility analysis (eq. 4), can be matched with the utility function of LFFRM (eq. 5). As a result, given $\alpha$ and $\beta$ values, it is possible identifying $\mathrm{w}_{l}$ and $\mathrm{w}_{\mathrm{f}}$ values by eq. 5 .

For $t>0$, evaluating the generic working cycle (i), the values of $t_{x}, F(t)$, and $F_{\max }$ can be identified in accordance with the dual-phase approach and LFFRM (eqs. 2, 8-9). Since $\mathrm{w}_{\mathrm{l}}$ and $\mathrm{w}_{\mathrm{f}}$ parameters are known (their values do not change over time), the model developed allows identifying the human error utility function $(\mathrm{U}(\mathrm{t}))$ and the human error probability $(\operatorname{HEP}(\mathrm{t}))$ over time (eqs. 5-10).

$$
\begin{aligned}
& \mathrm{F}\left(\mathrm{t}_{\mathrm{i}}\right)=\mathrm{R}\left(\mathrm{t}_{\mathrm{i}-1}\right)+\left(1-\mathrm{R}\left(\mathrm{t}_{\mathrm{i}-1}\right)\right)\left(1-\mathrm{e}^{\lambda \mathrm{t}_{\mathrm{i}}}\right) \\
& \mathrm{R}\left(\mathrm{t}_{\mathrm{i}}\right)=\mathrm{F}\left(\mathrm{t}_{\mathrm{i}-1}\right) \mathrm{e}^{-\mu \tau_{\mathrm{i}}}
\end{aligned}
$$

$\operatorname{HEP}(\mathrm{t})=10 \mathrm{E}(6 \log (\mathrm{U}(0)))$
Even if the model is based on approaches that consider both cognitive and motor tasks, it is intended for application on high-cognitive content tasks (e.g. visual inspections, vigilance tasks, scheduling activities, complex manually assembled, etc.). In the model, motor aspects are also taken into account since they are part of tasks independently from their cognitive load. Consistently, cognitive learning constant $\left(b_{c}\right)$ values greater than twice of the motor learning constant $\left(\mathrm{b}_{\mathrm{m}}\right)$ have been considered. This is consistent with cognitive-oriented tasks, characterized by high values of the LR. [28].

Finally, it is possible to claim that the model application allows identifying the HEP values for each t-values, considering the type of task performed, the worker characteristic, the work environment as well as the effect due to learning-forgetting and fatigue-recovery phenomena.

\section{Results and Discussion}

A numerical case study is developed in order to evaluate the effectiveness of the model. Consistently with this target, three different work break schedules (WBSs) are considered (fig. 2). The WBSs, identified with three different IDs (i.e. (i), (ii), and (iii)), are characterized by different length and frequency of rests in the work shift, but in all cases the cumulated work and break time for each work shift is the same (work-time 460 [min]; rest-time 50 [min]).

In order to compare the results of the model with an experimental case, the tasks considered are referred to an assembly of intake manifold, activity already evaluated by [24]. Tasks details and output of the Multi-attribute utility analysis $(t=0)$ are showed in tab. 2.

The values assumed for tasks and worker's characteristics (tab. 2) are estimated in accordance with

\section{ID WBS}

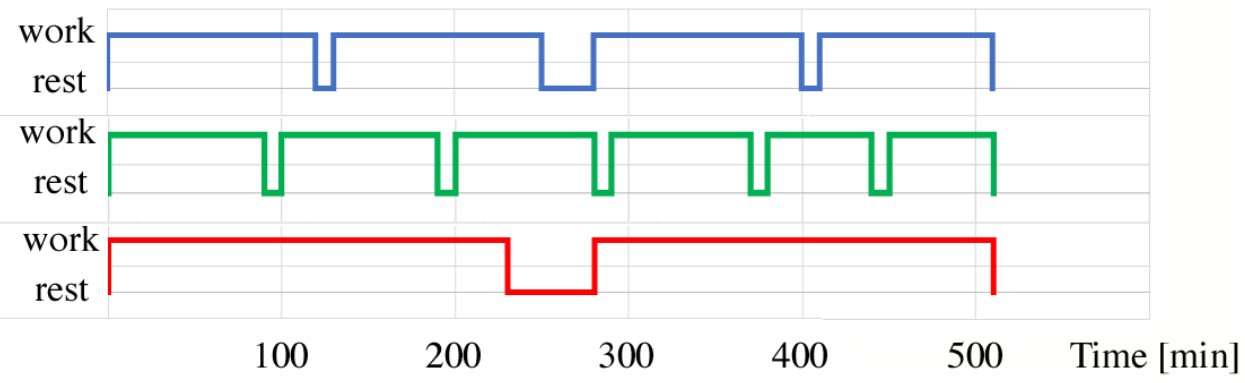

Figure 2. Work and rest time, in the work shift, of WBSs evaluated 
the experiments of Dar-el et al. (1995), in which the manual assembly operations were performed by graduate and under-graduate workers with an age ranging from 22 to 34 years.

On the basis of the input parameters, the model allows identifying the dynamic value of HEP over time (see fig. 3) by varying the WBS. It is possible to observe that the HEP\% is maximum, for all WBSs, at the beginning of the work shift (fig. 2). This value is consistent with results of multi-attribute utility analysis and does not depend on learning and/or fatigue effects. A decrease of HEP\% is observed in the first part of the work shift (around 100 minutes), since in this time period the worker's performance is mainly affected by learning phenomenon. In other words, in the first part of the work shift the worker, by optimizing the tasks required for parts assembly, is able to reduce the task completion time. After that, once the tasks execution time is not further improvable ('benefit' due to learning effects are heavily reduced), the worker performance is mainly affected by the fatigue phenomenon and the HEP\% increases over time. Under these conditions, the length and the frequency of breaks allow modifying the HEP\% variation over time. In particular, the results of the model showed that if on one hand the breaks reduce the HEP\%, on the other hand the recovery effect decreases during the work shift. When the fatigue effect prevails on the worker performance, the HEP\% tends to faster growth after each break. Consistently with this observation, the 'benefit' due to recovery time, i.e. the reduction of HEP\% during the breaks, is less effective over time. The evidence of this effect is showed by the increase of the curve slope after each break in fig. 3.

As far as concern the three WBSs considered (tab. 3), the lowest average HEP\% (during the work shift) is observed in case (i), although the standard deviation in case (ii) is smaller than other WBSs.

Table 2. Model input parameters

\begin{tabular}{|c|c|c|c|c|c|c|c|}
\hline \multicolumn{4}{|c|}{ Tasks and Worker characteristics } & \multicolumn{4}{|c|}{ Multi-attribute utility analysis $(\mathrm{t}=0)$} \\
\hline $\mathrm{T} 1[\mathrm{~s}]$ & 240 & $\lambda$ & 0.2 & $u_{x}$ & 0.536 & $\mathrm{w}_{\mathrm{x}}$ & 0.429 \\
\hline$a$ & 0.8 & $\mu$ & 0.5 & $\mathrm{u}_{\mathrm{y}}$ & 0.494 & $w_{y}$ & 0.143 \\
\hline \multirow[t]{3}{*}{$\beta$} & 0.8 & $\mathrm{t}_{\mathrm{c}}^{(1)} / \mathrm{t}_{\mathrm{m}}^{(1)}$ & 3.605 & $\mathrm{u}_{\mathrm{z}}$ & 0.506 & $\mathrm{w}_{\mathrm{z}}$ & 0.429 \\
\hline & & $b_{c}$ & 0.521 & & & & \\
\hline & & $b_{m}$ & 0.186 & & & & \\
\hline
\end{tabular}

Task description: the part to be assembled is unloaded from a trolley and a visually inspection for proper installation of all components is accomplished, then eight captive fasteners and one manifold absolute pressure sensor (to be placed to lower shell) are manually assembled. Finally, part is placed on transfer chute.

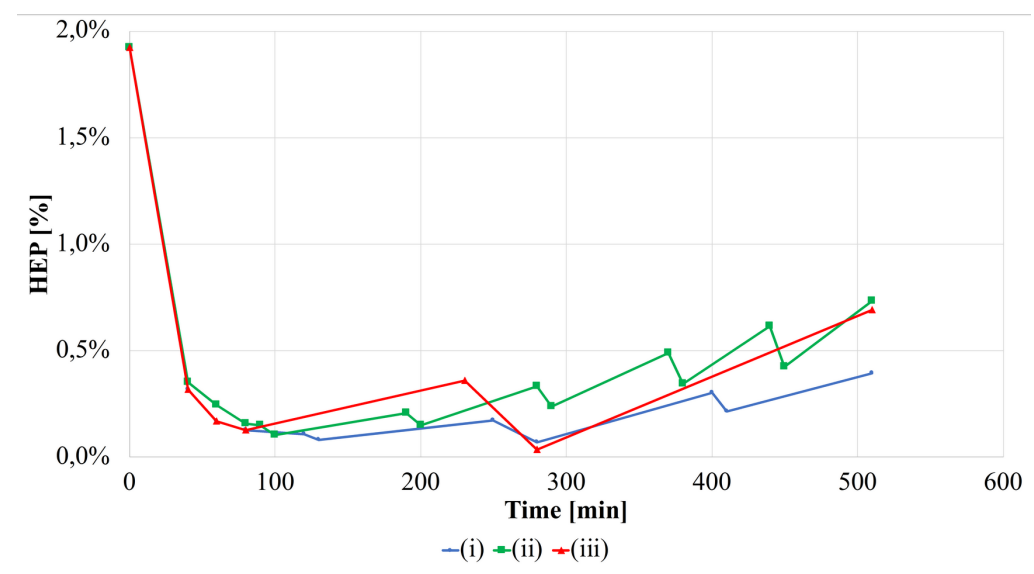

Figure 3. HEP\% curve over time, adopting different WBS 
Table 3. Model output parameters

\begin{tabular}{cccc}
\hline \multicolumn{3}{c}{ ID WBS } \\
\hline & (i) & (ii) & (iii) \\
Average HEP [\%] & 0.353 & 0.431 & 0.518 \\
St. Dev & $5.23 \mathrm{E}-3$ & $4.51 \mathrm{E}-3$ & $6.57 \mathrm{E}-3$ \\
\hline
\end{tabular}

Changing the input parameters (e.g. severity of task to be performed, speed of recovery, worker's learning rate, etc.) as well varying the frequency and the duration and the numbers of the breaks, the model allows identifying in every cases the dynamic variability of the HEP \% and the time window (in the work shift) characterized by highest/lowest worker's reliability values.

The results obtained are consistent with the experimental case proposed by Elmaraghy et al. (2008). Moreover, the trend of HEP\% is consistent with results of the application of LFFRM in an experimental case (Givi et al., 2015). The WBS identified as optimal by the model is in accordance with recently studies on work-rest policies, aiming at improving the workers' well-being [29].

\section{Conclusion}

Results of the study carried out showed that it is possible to evaluate the HEP over time on the basis of task, worker and workplace characteristics. The approach adopted allows considering the effect of fatigue and of recovery on HEP due to breaks in the work-shift. The results achieved are in accordance with existing evaluations and show the effect of different WBS schedule on the HEP variability over time. Considering the three research questions introduced in the first section, it is possible to claim that the model developed allows to predict the error probability in cognitive task varying worker's and task's characteristics and WBS adopted. The jointly adoption of multi-attribute utility analysis and LFFRM model allows to consider both 'time-dependent' aspects (e.g. learning, forgetting, fatigue, etc.) and aspects independently on time (e.g. task characteristics, workplace design, etc.) providing a dynamic evaluation of HEP over time. Finally, the model is easy to be implemented when compared with existing models in scientific literature. In the present study a simple VBA routine has been adopted to estimate the HEP\% over time. The reliability and effectiveness of the model has been tested by applying it in an industrial case, already faced in other scientific works. The comparison showed that the results obtained by the model defined are consistent with the results ob- tained in other experimental case studies.

The approach developed is focused on cognitive, rather than physical, tasks, therefore the flexibility of the model could be improved including the effect due to physical efforts on HEP, in order to extend the adoption of the method proposed to more complex scenarios. Moreover, specific guidelines or database could be required in order to support the user in identifying the proper values to be assigned to each input parameters depending on the case under investigation.

\section{Funding}

The research has been supported by the Italian Ministry of University and Research under the grant Research Project of National Interest (PRIN-2017) "Smart Operators 4.0 based on Simulation for Industry and Manufacturing Systems”.

\section{References}

[1] S.P. Whalley, "Minimising the cause of Human Error," in 10th Advances in Reliability Technology Symposium, 1988, pp. 114-128, doi: 10.1007/978-94-009-1355-4_11.

[2] M. Gattullo, A. Evangelista, A.E. Uva, M. Fiorentino, A. Boccaccio, V.M. Manghisi, "Exploiting Augmented Reality to Enhance Piping and Instrumentation Diagrams for Information Retrieval Tasks in Industry 4.0 Maintenance," in Virtual Reality and Augmented Reality. EuroVR 2019, vol 11883, doi: 10.1007/978-3-030-31908-3_11.

[3] A. Kujawińska, and K. Vogt, "Human factors in visual quality control," Management and Production Engineering Review, vol. 6, no. 2, pp. 25-31, Jun. 2015, doi: 10.1515/mper-2015-0013.

[4] R. Carli, M. Dotoli, N. Epicoco, B. Angelico, B, and A. Vinciullo, "Automated evaluation of urban traffic congestion using bus as a probe," IEEE International Conference on Automation Science and Engineering (CASE), 2015, doi: 10.1109/CoASE.2015.7294224.

[5] P. Purpura "The History of Security and Loss Prevention: A Critical Perspective," Security and Loss Prevention, pp. 3-20, 2008, doi:10.1016/B978-(0-08-055400-6.50007-8.

[6] International Air Transport Association, "Safety Report 2017 - 54th edition." [Online]. Available: https://aviation-safety.net/airlinesafety/industry/reports/IA TA-safety-report-2017.pdf [Accessed: 8-March-2019].

[7] IChemE, "Loss Preventio Bulletin." [Online]. Available: https://www.icheme.org/media/1278/lpb251_digimag.pdf. [Accessed: 14-March-2019].

[8] R. Carli, M. Dotoli, and R. Pellegrino, "Multi-criteria decision-making for sustainable metropolitan cities assessment," Journal of Environmental Management, vol. 226, no. 15, pp. 46-61, Nov. 2018, doi: 10.1016/j.jenvman.2018.07.075.

[9] I.M. Dragan, and A. ISaic-Maniu, "The reliability of the human factor," Procedia Economics and Finance, vol. 15, pp. 1486-1494, 2014 doi: 10.1016/S2212-5671(14)00615-7.

[10] A. E. Uva, M. Fiorentino, V.M. Manghisi, A. Boccaccio, S. Debernardis, M. Gattullo, G. Monno, "A User-Centered 
Framework for Designing Midair Gesture Interfaces," IEEE Transactions on Human-Machine Systems, vol. 49, no.5,pp.421-429, 2019, doi: 10.1109/THMS.2019.2919719.

[11] G. Intranuovo, N. Schiavulli, D. Cavone, F. Birtolo, P. Cocco, L. Vimercati, L. Macinagrossa, A. Giordano, T. Perrone, G. Ingravallo, P. Mazza, M. Strusi, C. Spinosa, G. Specchia, and G.M. Ferri, "Assessment of DNA damages in lymphocytes of agricultural workers exposed to pesticides by comet assay in a cross-section al study," Biomarkers, vol. 23, no. 5, pp. 462-473, 2018, doi: 10.1080/1354750X.2018.1443513.

[12] E. Calixto, G.B.A. Lima, and R.A. Firmino, "Comparing SLIM, SPAR-H and Bayesian Network Methodologies," Open Journal of Safety Science and Technology, vol. 3, no. 2, Jun. 2013, doi:10.4236/ojsst.2013.32004.

[13] M. Bevilacqua, and F.E. Ciarapica, "Human factor risk management in the process industry: A case study, "Reliability Engineering and System Safety, vol. 169, no. C, pp. 149-159, 2018, doi: 10.1016/j.ress.2017.08.013.

[14] Health and safety executive, "Review of human reliability assessment methods." [Online]. Available: http://www.hse. gov.uk/research/rrhtm/rr679.htm.[Accessed: 14-May-2019].

[15] P. Trucco, and M.C. Leva, "A probabilistic cognitive simulator for HRA studies (PROCOS), Reliability Engineering and System Safety, vol. 92, no. 8, pp. 11171130, Aug. 2007, doi: 10.1016/j.ress.2006.06.003.

[16] S. Zhang, W. He, D. Chen, J. Chu, and H. Fan, "A dynamic human reliability assessment approach for manned submersibles using PMV-CREAM," International Journal of Naval Architecture and Ocean Engineering, vol. 11, no. 2, pp. 782-795, Jul. 2019, doi: 10.1016/j.ijnaoe.2019.03.002.

[17] G.A. Shirali, T. Hosseinzadeh, K.A. Angali, and S.R.N. Kalhori, "Modifying a method for human reliability assessment based on CREAM-BN: A case study in control room of a petrochemical plant," MethodsX, vol. 6, pp. 300315, 2019, doi: 10.1016/j.mex.2019.02.008.

[18] S. Rangra, M. Sallak, W. Schön, and F. Vanderhaegen, "Human reliability assessment under uncertainty -towards a formal method," Procedia Manufacturing, vol. 3, pp. 32303237, 2015, doi: 10.1016/j.promfg.2015.07.874.

[19] S. Hosseini, R. Carli, and M. Dotoli, "Robust Day-Ahead Energy Scheduling of a Smart Residential User Under Uncertainty," 18th European Control Conference (ECC), 2018, doi: 10.23919/ECC.2019.8796182.

[20] N. Margiotta, G. Avitabile, G. and G. Coviello, "A wearable wireless system for gait analysis for early diagnosis of Alzheimer and Parkinson disease" 5th International Conference on Electronic Devices, 2016, Systems and Applications (ICEDSA), doi: 10.1109/ICED SA.2016.7818553.

[21] M.M. Abaei, R. Abbassi, V. Garaniya, E. Arzaghi, and A.B. Toroody "A dynamic human reliability model for marine and offshore operations in harsh environments," Ocean Engineering, vol. 173, no. 1, pp. 90-97, Feb. 2019, doi: 10.1016/j.oceaneng.2018.12.032.

[22] R. Islam, R. Abbassi, V. Garaniya, and F. Khan, "Development of a human reliability assessment technique for the maintenance procedures of marine and offshore operations," Journal of Loss Prevention in the Process Industries, vol. 50, pp. 416-428, Nov. 2017, doi: 10.1016/j.jlp.2017.10.015.

[23] D. Falcone, F. De Felice, A. Petrillo, and A. Silvestri, "An Experimental Study on Developing a Cognitive Model for Human Reliability Analysis," in Theory and Application on Cognitive Factors and Risk Management, IntechOpen, 2017, doi: 10.5772/intechopen.69230.

[24] W.H. Elmaraghy, O.A. Nada, and H.A. Elmaraghy, "Quality prediction for reconfigurable manufacturing systems via human error modelling," International Journal of Computer Integrated Manufacturing, vol. 21, no. 5, pp. 584-598, Jun. 2008, doi: 10.1080/09511920701233464.

[25] Z.S. Givi, M.Y. Jaber, and W.P. Neumann, "Modelling worker reliability with learning and fatigue," Applied Mathematical Modelling, vol. 39, no. 17, pp. 5186-5199, 2015, doi: 10.1016/j.apm.2015.03.038.

[26] T.P. Wright, "Factors affecting the cost of airplanes", Journal of Aeronautical Sciences, vol. 3, pp. 122-128, 1936.

[27] E.M. Dar-El, K. Ayas, and I. Gilad, "A dual-phase model for the individual learning process in industrial tasks," IIE Transactions, vol. 27, no. 3, pp. 265-271, Apr. 2007, doi: 10.1080/07408179508936740.

[28] E.M. Dar-El, "HUMAN LEARNING: From Learning Curves to Learning Organizations," Springer Science \& Business Media.

[29] H. Zacher, H.A. Brailsford, and S.L. Parker, "Micro-breaks matter: A diary study on the effects of energy management strategies on occupational well-being," Journal of Vocational Behavior, vol. 85, no. 3, pp. 287-297, Dec. 2014, doi: $10.1016 /$ j.jvb.2014.08.005. 